\title{
Left residuated operators induced by posets with a unary operation
}

\author{
Ivan Chajda ${ }^{1} \cdot$ Helmut Länger ${ }^{1,2}$
}

Published online: 6 May 2019

(c) The Author(s) 2019

\begin{abstract}
The concept of operator left residuation has been introduced by the authors in their previous paper (Chajda and Länger in Asian Eur J Math 11:1850097, 2018). Modifications of so-called quantum structures, in particular orthomodular posets, like pseudo-orthomodular, pseudo-Boolean and Boolean posets are investigated here in order to show that they are operator left residuated or even operator residuated. In fact, they satisfy more general sufficient conditions for operator residuation assumed for bounded posets equipped with a unary operation. It is shown that these conditions may be also necessary if a generalized version using subsets instead of single elements is considered. The above-listed posets can serve as an algebraic semantics for the logic of quantum mechanics in a broad sense. Moreover, our approach shows connections to substructural logics via the considered residuation.
\end{abstract}

Keywords Operator residuation - Operator left adjointness · Boolean poset · Pseudo-Boolean poset · Pseudo-orthomodular poset $\cdot$ Generalized operator residuation

\section{Introduction}

It was shown by Birkhoff and von Neumann (1936) and, independently, by Husimi (1937) that orthomodular lattices can serve as an algebraic semantics of the logic of quantum mechanics. Namely, the class of event-state systems in quantum mechanics is usually identified with the set of projection operators on a Hilbert space $\mathbf{H}$ and this set is in a bijective correspondence with the set of all closed linear subspaces of $\mathbf{H}$. However, certain doubts concerning the relevance of this representation arose when it was shown that the class of orthomodular lattices arising in this way does not generate the variety of orthomodular lattices. In other words, there exist orthomodular lattices which do not repre-

Communicated by A. Di Nola.

Helmut Länger

helmut.laenger@tuwien.ac.at

Ivan Chajda

ivan.chajda@upol.cz

1 Department of Algebra and Geometry, Faculty of Science, Palacký University Olomouc, 17. Listopadu 12, 77146 Olomouc, Czech Republic

2 Institute of Discrete Mathematics and Geometry, Faculty of Mathematics and Geoinformation, TU Wien, Wiedner Hauptstraße 8-10, 1040 Vienna, Austria sent a physical system in the logic of quantum mechanics. The reason is that some equational properties of event-state systems are not fairly reflected by the proposed mathematical abstraction. This was the reason for alternative approaches (see, e.g., Giuntini et al. (2016, 2017)). In particular, an algebraic semantics for the logic of quantum mechanics was found among orthomodular posets and their modifications.

Orthomodular lattices have similar properties as algebraic structures used for substructural logics (see, e.g., Galatos et al. (2007)). The authors proved in Chajda and Länger $(2017 \mathrm{a}, \mathrm{b})$ that every orthomodular lattice can be converted into a so-called left residuated l-groupoid. They showed in Chajda and Länger (submitted) that this result can be easily extended to a certain class of bounded lattices with a unary operation which, of course, contains the variety of orthomodular lattices. Hence, the natural question arises if this approach can be extended to ordered sets with a unary operation. For this purpose, so-called residuated operators were introduced in Chajda and Länger (2018) and several classes of ordered sets with a unary operation turned out to be operator residuated. A prominent role among these posets play the so-called pseudo-orthomodular posets which are a direct generalization of orthomodular lattices, but serve also as good candidates for an algebraic semantics of the logic of quantum mechanics. And 
again, there arises the natural question if the posets listed in Chajda and Länger (2018) really exhaust all possible cases.

The aim of the present paper is to provide several simple conditions under which a bounded poset with a unary operation can be organized into an operator left residuated poset. Similarly as it was done for lattices in Chajda and Länger (submitted), we ask whether these conditions are not only sufficient but also necessary. It is shown that if subsets instead of single elements are considered, then these generalized conditions characterize the class of posets which can be converted into operator residuated ones.

\section{Adjointness of operators}

Recall from Birkhoff and von Neumann (1936) that an orthomodular lattice is a bounded lattice $\left(L, \vee, \wedge,{ }^{\prime}, 0,1\right)$ with a unary operation' which is a complementation and an antitone involution (see, e.g., Kalman 1958) satisfying the orthomodular law

$x \leq y$ implies $x=y \wedge\left(x \vee y^{\prime}\right)$

or, equivalently,

$x \leq y$ implies $y=x \vee\left(y \wedge x^{\prime}\right)$.

A left residuated lattice (or integral l-groupoid in the terminology of Galatos et al. 2007) is a bounded lattice $\mathbf{L}=$ $(L, \vee, \wedge, \odot, \rightarrow, 0,1)$ with two more binary operations $\odot$ and $\rightarrow$ satisfying

$x \odot 1 \approx 1 \odot x \approx x$

$x \odot y \leq z$ if and only if

$x \leq y \rightarrow z$ (the so-called left adjointness).

Here "L satisfies the identity $x \odot 1 \approx x$ " means that " $x \odot$ $1=x$ for all $x \in L "$. We put $x^{\prime}:=x \rightarrow 0$. If $\odot$ is, moreover, commutative, then we call the previous property simply adjointness.

Let $(P, \leq)$ be a poset, $a, b \in P$ and $A, B \subseteq P$. Then, we define

$L(A):=\{x \in P \mid x \leq y$ for all $y \in A\}$,

$U(A):=\{x \in P \mid x \geq y$ for all $y \in A\}$.

Instead of $L(\{a, b\}), L(A \cup\{b\}), L(A \cup B)$, we write $L(a, b), L(A, b), L(A, B)$, respectively. Similar expressions are treated analogously. Moreover, if ' is a unary operation on $P$, then $A^{\prime}:=\left\{x^{\prime} \mid x \in A\right\}$.
It was shown by the authors in Chajda and Länger $(2017 \mathrm{a}, \mathrm{b})$ that taking

$x \odot y:=\left(x \vee y^{\prime}\right) \wedge y$,

$x \rightarrow y:=(y \wedge x) \vee x^{\prime}$

in an orthomodular lattice $\mathbf{L}=\left(L, \vee, \wedge,{ }^{\prime}, 0,1\right)$ yields a left residuated lattice $(L, \vee, \wedge, \odot, \rightarrow, 0,1)$ where $x^{\prime}=x \rightarrow 0$ coincides with the complementation in $\mathbf{L}$.

However, as shown in Chajda and Länger (2018), if $\left(P, \leq,{ }^{\prime}, 0,1\right)$ is an orthomodular poset (or even a Boolean poset), then such operations $\odot$ and $\rightarrow$ need not exist. In order to avoid these complications, we study bounded ordered sets with a unary operation. We introduced in Chajda and Länger (2018) the following notion:

Definition 2.1 An operator left residuated poset is an ordered seventuple $\mathbf{P}=\left(P, \leq,^{\prime}, M, R, 0,1\right)$ where $\left(P, \leq,^{\prime}, 0,1\right)$ is a bounded poset with a unary operation and $M$ and $R$ are mappings from $P^{2}$ to $2^{P}$ satisfying the following conditions for all $x, y, z \in P$ :

(i) $M(x, 1)=M(1, x)=L(x)$,

(ii) $M(x, y) \subseteq L(z)$ if and only if $L(x) \subseteq R(y, z)$,

(iii) $R(x, 0)=L\left(x^{\prime}\right)$.

Condition (ii) is called operator left adjointness. If $M$ is commutative, then (ii) is called operator adjointness only and $\mathbf{P}$ is called an operator residuated poset.

In Chajda and Länger (2018), the definition contains one more condition which, however, follows from (i) and (ii) (see the following lemma).

Lemma 2.2 Everyoperator left residuated poset $\left(P, \leq,{ }^{\prime}, M\right.$, $R, 0,1)$ satisfies the following condition for all $x, y \in P$ :

$R(x, y)=P$ if and only if $x \leq y$.

Proof For $x, y \in P$, the following are equivalent:

$$
\begin{aligned}
R(x, y) & =P, \\
L(1) & \subseteq R(x, y), \\
M(1, x) & \subseteq L(y), \\
L(x) & \subseteq L(y), \\
x & \leq y .
\end{aligned}
$$

For posets $\left(P, \leq,^{\prime}\right)$ with a unary operation, we define the following two conditions:

$$
\begin{aligned}
& L(x) \subseteq L\left(U\left(L\left(U\left(x, y^{\prime}\right), y\right), y^{\prime}\right)\right) \text { for all } x, y \in P, \\
& L\left(U\left(L(x, y), y^{\prime}\right), y\right) \subseteq L(x) \text { for all } x, y \in P,
\end{aligned}
$$


and the following two mappings from $P^{2}$ to $2^{P}$ :

$$
\begin{aligned}
M(x, y) & :=L\left(U\left(x, y^{\prime}\right), y\right) \text { for all } x, y \in P, \\
R(x, y) & :=L\left(U\left(L(y, x), x^{\prime}\right)\right) \text { for all } x, y \in P .
\end{aligned}
$$

Lemma 2.3 Let $\left(P, \leq,^{\prime}\right)$ be a poset with a unary operation and $M$ and $R$ defined by (3) and (4), respectively. Then, (1) implies $M(x, y) \subseteq L(z) \Rightarrow L(x) \subseteq R(y, z)$ and (2) implies $L(x) \subseteq R(y, z) \Rightarrow M(x, y) \subseteq L(z)$.

Proof Assume $a, b, c \in P$. If (1) and $M(a, b) \subseteq L(c)$, then

$$
\begin{aligned}
L(a) & \subseteq L\left(U\left(L\left(U\left(a, b^{\prime}\right), b\right), b^{\prime}\right)\right) \\
& =L\left(U\left(L\left(U\left(a, b^{\prime}\right), b\right) \cap L(b), b^{\prime}\right)\right) \\
& =L\left(U\left(M(a, b) \cap L(b), b^{\prime}\right)\right) \subseteq L\left(U\left(L(c) \cap L(b), b^{\prime}\right)\right) \\
& =L\left(U\left(L(c, b), b^{\prime}\right)\right)=R(b, c) .
\end{aligned}
$$

If (2) and $L(a) \subseteq R(b, c)$, then

$$
\begin{aligned}
M(a, b) & =L\left(U\left(a, b^{\prime}\right), b\right)=L\left(U(a) \cap U\left(b^{\prime}\right), b\right) \\
& =L\left(U(L(a)) \cap U\left(b^{\prime}\right), b\right) \\
& \subseteq L\left(U(R(b, c)) \cap U\left(b^{\prime}\right), b\right) \\
& =L\left(U\left(L\left(U\left(L(c, b), b^{\prime}\right)\right)\right) \cap U\left(b^{\prime}\right), b\right) \\
& =L\left(U\left(L(c, b), b^{\prime}\right) \cap U\left(b^{\prime}\right), b\right) \\
& =L\left(U\left(L(c, b), b^{\prime}\right), b\right) \subseteq L(c) .
\end{aligned}
$$

Definition 2.4 Recall (e.g., from Chajda and Rachůnek $1989)$ that a distributive poset is a poset $(P, \leq)$ satisfying one of the following equivalent identities:

$L(U(x, y), z) \approx L(U(L(x, z), L(y, z)))$,

$U(L(x, y), z) \approx U(L(U(x, z), U(y, z)))$.

A poset with complementation is an ordered quintuple $\mathbf{P}=$ $\left(P, \leq,{ }^{\prime}, 0,1\right)$ such that $(P, \leq, 0,1)$ is a bounded poset and ${ }^{\prime}$ is a unary operation on $P$ satisfying the following conditions for all $x, y \in P$ :

(i) $L\left(x, x^{\prime}\right) \approx\{0\}$ and $U\left(x, x^{\prime}\right) \approx\{1\}$,

(ii) $x \leq y$ implies $y^{\prime} \leq x^{\prime}$,

(iii) $\left(x^{\prime}\right)^{\prime} \approx x$.

As mentioned in Introduction, we introduce several kinds of posets with complementation which generalize orthomodular lattices.

The poset $\mathbf{P}$ with complementation is called a Boolean poset if $(P, \leq)$ is distributive. Of course, every Boolean algebra is a Boolean poset, but there are interesting examples of Boolean posets which are not lattices (see, e.g., Chajda and Länger (submitted)). In every case, Boolean posets are orthomodular posets, and hence, they can be considered as quantum structures.

The poset $\mathbf{P}$ with complementation is called a pseudoBoolean poset if it satisfies one of the following equivalent identities:

$L\left(U(x, y), y^{\prime}\right) \approx L\left(x, y^{\prime}\right)$,

$U\left(L(x, y), y^{\prime}\right) \approx U\left(x, y^{\prime}\right)$.

The identities defining a pseudo-Boolean poset are generalizations of the Sasaki projection and the Sasaki hook. Pseudo-Boolean posets are certain generalization of Boolean ones, but they are closely connected to the following posets. The operators $M$ and $R$ defined by

$$
\begin{aligned}
M(x, y) & :=L(x, y) \text { for all } x, y \in P, \\
R(x, y) & :=L\left(U\left(y, x^{\prime}\right)\right) \text { for all } x, y \in P
\end{aligned}
$$

mimic meet and material implication, respectively. This gives these posets their pseudo-Boolean character.

The poset $\mathbf{P}$ with complementation is called a pseudoorthomodular poset if it satisfies one of the following equivalent identities:

$L\left(U\left(L(x, y), y^{\prime}\right), y\right) \approx L(x, y)$,

$U\left(L\left(U(x, y), y^{\prime}\right), y\right) \approx U(x, y)$.

It is evident that pseudo-orthomodular posets are generalizations of orthomodular lattices. Namely, if a poset $\left(P, \leq,{ }^{\prime}, 0,1\right)$ with complementation is a lattice satisfying these identities, then $U(x, y) \approx U(x \vee y)$ and $L(x, y) \approx$ $L(x \wedge y)$. Thus, our equalities yield

$$
\begin{aligned}
& L\left(\left((x \wedge y) \vee y^{\prime}\right) \wedge y\right) \approx L\left((x \wedge y) \vee y^{\prime}, y\right) \\
& \quad \approx L\left(U\left((x \wedge y) \vee y^{\prime}\right), y\right) \approx L\left(U\left(x \wedge y, y^{\prime}\right), y\right) \\
& \quad \approx L\left(U\left(L(x \wedge y), y^{\prime}\right), y\right) \approx L\left(U\left(L(x, y), y^{\prime}\right), y\right) \\
& \quad \approx L(x, y) \approx L(x \wedge y)
\end{aligned}
$$

whence

$\left((x \wedge y) \vee y^{\prime}\right) \wedge y \approx x \wedge y$

which is the orthomodular law. Hence, these posets can serve as an algebraic semantics of the logic of quantum mechanics. The advantage of this concept is that we need not assume $x \leq y$ as in the definition of orthomodular posets.

It is easy to see that every Boolean poset is pseudoBoolean and every pseudo-Boolean poset is pseudoorthomodular (cf. Chajda and Länger 2018).

An example of a pseudo-orthomodular poset which is neither Boolean nor orthomodular is depicted in Fig. 1. It is not 


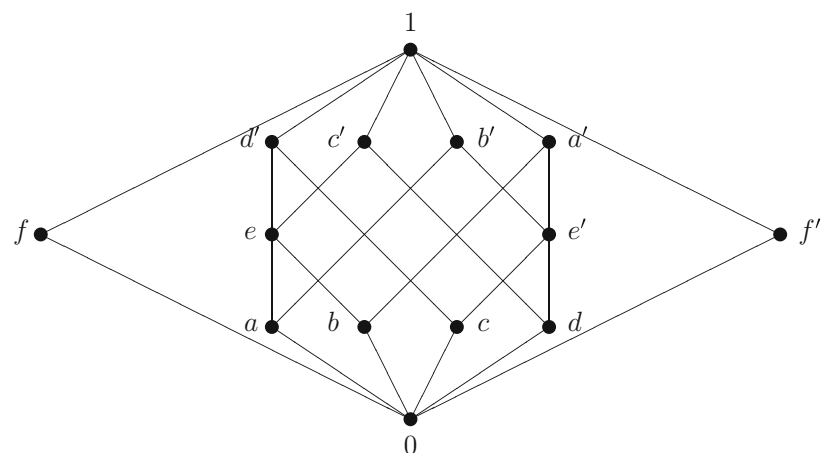

Fig. 1 A pseudo-orthomodular poset

orthomodular because, e.g., $b \leq c^{\prime}$, but $b \vee c$ does not exist. It is evident that it is not Boolean because it is not distributive.

Theorem 2.5 Let $\left(P, \leq,{ }^{\prime}, 0,1\right)$ be a bounded poset with a unary operation satisfying both conditions (1) and (2) and the identity $1^{\prime} \approx 0$ and $M$ and $R$ defined by (3) and (4), respectively. Then, $\left(P, \leq,{ }^{\prime}, M, R, 0,1\right)$ is an operator left residuated poset.

Proof (i) $M(x, 1) \approx L\left(U\left(x, 1^{\prime}\right), 1\right) \approx L\left(U\left(x, 1^{\prime}\right)\right) \approx$ $L(U(x, 0)) \approx L(U(x)) \approx L(x)$,

$M(1, x) \approx L\left(U\left(1, x^{\prime}\right), x\right) \approx L(1, x) \approx L(x)$,

(ii) Follows from Lemma 2.3,

(iii) $R(x, 0) \approx L\left(U\left(L(0, x), x^{\prime}\right)\right) \approx L\left(U\left(0, x^{\prime}\right)\right) \approx$ $L\left(U\left(x^{\prime}\right)\right) \approx L\left(x^{\prime}\right)$.

We show that the posets mentioned above are among those assumed in Theorem 2.5.

Example 2.6 Every pseudo-orthomodular poset satisfies both (1) and (2). This can be seen as follows: If $\left(P, \leq,{ }^{\prime}, 0,1\right)$ is a pseudo-orthomodular poset and $a, b \in P$, then

$L(a)=L(U(a)) \subseteq L\left(U\left(a, b^{\prime}\right)\right)=L\left(U\left(L\left(U\left(a, b^{\prime}\right), b\right), b^{\prime}\right)\right)$,

$L\left(U\left(L(a, b), b^{\prime}\right), b\right)=L(a, b) \subseteq L(a)$.

For posets $\left(P, \leq,^{\prime}\right)$ with a unary operation, we define the following two conditions:

$L(x) \subseteq L\left(U\left(L(x, y), y^{\prime}\right)\right)$ for all $x, y \in P$,

$L\left(U\left(x, y^{\prime}\right), y\right) \subseteq L(x)$ for all $x, y \in P$

and the following two mappings from $P^{2}$ to $2^{P}$ :

$M(x, y):=L(x, y)$ for all $x, y \in P$,

$R(x, y):=L\left(U\left(y, x^{\prime}\right)\right)$ for all $x, y \in P$.

Observe that $M$ is commutative.
Lemma 2.7 Let $\left(P, \leq,{ }^{\prime}\right)$ be a poset with a unary operation and $M$ and $R$ defined by (7) and (8), respectively. Then, (5) implies $M(x, y) \subseteq L(z) \Rightarrow L(x) \subseteq R(y, z)$ and (6) implies $L(x) \subseteq R(y, z) \Rightarrow M(x, y) \subseteq L(z)$.

Proof Assume $a, b, c \in P$. If (5) and $M(a, b) \subseteq L(c)$, then

$$
\begin{aligned}
L(a) & \subseteq L\left(U\left(L(a, b), b^{\prime}\right)\right)=L\left(U(L(a, b)) \cap U\left(b^{\prime}\right)\right) \\
& =L\left(U(M(a, b)) \cap U\left(b^{\prime}\right)\right) \\
& \subseteq L\left(U(L(c)) \cap U\left(b^{\prime}\right)\right)=L\left(U(c) \cap U\left(b^{\prime}\right)\right) \\
& =L\left(U\left(c, b^{\prime}\right)\right)=R(b, c) .
\end{aligned}
$$

If (6) and $L(a) \subseteq R(b, c)$, then

$$
\begin{aligned}
M(a, b) & =L(a, b)=L(a) \cap L(b) \subseteq R(b, c) \cap L(b) \\
& =L\left(U\left(c, b^{\prime}\right)\right) \cap L(b)=L\left(U\left(c, b^{\prime}\right), b\right) \subseteq L(c) .
\end{aligned}
$$

Theorem 2.8 Let $\left(P, \leq,{ }^{\prime}, 0,1\right)$ be a bounded poset with a unary operation satisfying both conditions (5) and (6) and $M$ and $R$ defined by (7) and (8), respectively. Then, $\left(P, \leq,{ }^{\prime}, M, R, 0,1\right)$ is an operator residuated poset.

Proof (i) $M(x, 1) \approx L(x, 1) \approx L(x)$,

$M(1, x) \approx L(1, x) \approx L(x)$,

(ii) Follows from Lemma 2.7,

(iii) $R(x, 0) \approx L\left(U\left(0, x^{\prime}\right)\right) \approx L\left(U\left(x^{\prime}\right)\right) \approx L\left(x^{\prime}\right)$.

Since $M$ is commutative, $\left(P, \leq,^{\prime}, M, R, 0,1\right)$ is an operator residuated poset.

Again, pseudo-Boolean and hence also Boolean posets are among those posets assumed in Theorem 2.8 (see the following example).

Example 2.9 Every pseudo-Boolean poset satisfies (5) and (6). This can be seen as follows: If $\left(P, \leq,{ }^{\prime}, 0,1\right)$ is a pseudoBoolean poset and $a, b \in P$, then

$L(a)=L(U(a)) \subseteq L\left(U\left(a, b^{\prime}\right)\right)=L\left(U\left(L(a, b), b^{\prime}\right)\right)$,

$L\left(U\left(a, b^{\prime}\right), b\right)=L(a, b) \subseteq L(a)$.

Combining Theorems 2.5 and 2.8 and Examples 2.6 and 2.9, we conclude

Corollary 2.10 If $\left(P, \leq,{ }^{\prime}, 0,1\right)$ is a pseudo-Boolean poset and $M$ and $R$ are defined by (7) and (8), respectively, then $\left(P, \leq,^{\prime}, M, R, 0,1\right)$ is an operator residuated poset. If $\left(P, \leq,{ }^{\prime}, 0,1\right)$ is a pseudo-orthomodular poset satisfying the identity $1^{\prime} \approx 0$ and $M$ and $R$ are defined by (3) and (4), respectively, then $\left(P, \leq{ }^{\prime}, M, R, 0,1\right)$ is an operator left residuated poset.

It is well known that in a residuated lattice each of the operations $\odot$ and $\rightarrow$ determines the other one. We can prove a similar result also for the posets listed above. 
Proposition 2.11 (i) If $\left(P, \leq,{ }^{\prime}, 0,1\right)$ is a poset with complementation and $M$ and $R$ are defined by (3) and (4), respectively, then

$$
\begin{aligned}
L\left(\left(M\left(y^{\prime}, x\right)\right)^{\prime}\right) & \approx R(x, y), \\
L\left(\left(R\left(y, x^{\prime}\right)\right)^{\prime}\right) & \approx M(x, y) .
\end{aligned}
$$

(ii) If $(P, \leq, ', 0,1)$ is a poset with complementation and $M$ and $R$ are defined by (7) and (8), respectively, then

$$
\begin{aligned}
L\left(\left(M\left(y^{\prime}, x\right)\right)^{\prime}\right) & \approx R(x, y), \\
L\left(\left(R\left(y, x^{\prime}\right)\right)^{\prime}\right) & \approx M(x, y) .
\end{aligned}
$$

Proof (i)

$$
\begin{aligned}
L\left(\left(M\left(y^{\prime}, x\right)\right)^{\prime}\right) & \approx L\left(\left(L\left(U\left(y^{\prime}, x^{\prime}\right), x\right)\right)^{\prime}\right) \\
& \approx L\left(U\left(L(y, x), x^{\prime}\right)\right) \approx R(x, y), \\
L\left(\left(R\left(y, x^{\prime}\right)\right)^{\prime}\right) & \approx L\left(\left(L\left(U\left(L\left(x^{\prime}, y\right), y^{\prime}\right)\right)\right)^{\prime}\right) \\
& \approx L\left(U\left(L\left(U\left(x, y^{\prime}\right), y\right)\right)\right) \\
& \approx L\left(U\left(x, y^{\prime}\right), y\right) \\
& \approx M(x, y),
\end{aligned}
$$

(ii)

$$
\begin{aligned}
L\left(\left(M\left(y^{\prime}, x\right)\right)^{\prime}\right) & \approx L\left(\left(L\left(y^{\prime}, x\right)\right)^{\prime}\right) \approx L\left(U\left(y, x^{\prime}\right)\right) \\
& \approx R(x, y), \\
L\left(\left(R\left(y, x^{\prime}\right)\right)^{\prime}\right) & \approx L\left(\left(L\left(U\left(x^{\prime}, y^{\prime}\right)\right)\right)^{\prime}\right) \approx L(U(L(x, y))) \\
& \approx L(x, y) \approx M(x, y) .
\end{aligned}
$$

\section{A characterization of posets satisfying generalized operator residuation}

The conditions (5), (6) as well as (7), (8) which are formulated for variables can be expressed also for subsets of $P$ in the following way:

For posets $\left(P, \leq,^{\prime}\right)$ with a unary operation, we define the following two conditions:

$L(A) \subseteq L\left(U\left(L(A, B), B^{\prime}\right)\right)$ for all $A, B \subseteq P$,

$L\left(U\left(A, B^{\prime}\right), B\right) \subseteq L(A)$ for all $A, B \subseteq P$

and the following two binary operations on $2^{P}$ :

$$
\begin{aligned}
M(A, B) & :=L(A, B) \text { for all } A, B \subseteq P, \\
R(A, B) & :=L\left(U\left(B, A^{\prime}\right)\right) \text { for all } A, B \subseteq P .
\end{aligned}
$$

In case $A=\{x\}$ and $B=\{y\}$, we will write simply $M(x, y)$ and $R(x, y)$ as previously. Of course, taking singletons in (9) and (10) instead of $A$ and $B$ yields (5) and (6), respectively. Hence, the new conditions and definitions include the previous ones as a particular case. Also our definition of operator adjointness can be extended to subsets of $P$ as follows:

for all $A, B, C \subseteq P, M(A, B) \subseteq L(C)$ implies

$$
L(A) \subseteq R(B, C) \text {, }
$$

for all $A, B, C \subseteq P, L(A) \subseteq R(B, C)$ implies

$$
M(A, B) \subseteq L(C) \text {. }
$$

The ordered seventuple $\mathbf{P}=\left(P, \leq,{ }^{\prime}, M, R, 0,1\right)$ will be called a generalized operator left residuated poset if it satisfies (i) and (iii) of Definition 2.1 as well as (13) and (14), i.e., if for all $A, B, C \subseteq P$,

$M(A, B) \subseteq L(C)$ is equivalent to $L(A) \subseteq R(B, C)$

Condition (15) will be called generalized operator left adjointness. If $M$ is commutative, then (15) is called generalized operator adjointness only and $\mathbf{P}$ is called a generalized operator residuated poset. It is evident that taking singletons instead of $A, B, C$ in generalized operator adjointness, we obtain condition (ii) from Definition 2.1. Now we are able to prove a result analogous to Lemma 2.7.

Theorem 3.1 Let $\left(P, \leq,,^{\prime}\right)$ be a poset with a unary operation and $M$ and $R$ defined by (11) and (12), respectively. Then, (9) implies (13) are equivalent, and (10) implies (14) are equivalent.

Proof Assume $A, B, C \subseteq P$.

(9) $\Rightarrow(13)$ :

If $M(A, B) \subseteq L(C)$, then

$$
\begin{aligned}
L(A) & \subseteq L\left(U\left(L(A, B), B^{\prime}\right)\right)=L\left(U(L(A, B)) \cap U\left(B^{\prime}\right)\right) \\
& =L\left(U(M(A, B)) \cap U\left(B^{\prime}\right)\right) \\
& \subseteq L\left(U(L(C)) \cap U\left(B^{\prime}\right)\right) \subseteq L\left(U(C) \cap U\left(B^{\prime}\right)\right) \\
& =L\left(U\left(C, B^{\prime}\right)\right)=R(B, C) .
\end{aligned}
$$

$(10) \Rightarrow(14)$ :

If $L(A) \subseteq R(B, C)$, then

$$
\begin{aligned}
M(A, B) & =L(A, B)=L(A) \cap L(B) \subseteq R(B, C) \cap L(B) \\
& =L\left(U\left(C, B^{\prime}\right)\right) \cap L(B) \\
& =L\left(U\left(C, B^{\prime}\right), B\right) \subseteq L(C) .
\end{aligned}
$$

$(14) \Rightarrow(10)$ :

Any of the following assertions implies the next one: 


$$
\begin{aligned}
L\left(U\left(A, B^{\prime}\right)\right) & \subseteq L\left(U\left(A, B^{\prime}\right)\right), \\
L\left(U\left(A, B^{\prime}\right)\right) & \subseteq R(B, A), \\
M\left(U\left(A, B^{\prime}\right), B\right) & \subseteq L(A), \\
L\left(U\left(A, B^{\prime}\right), B\right) & \subseteq L(A) .
\end{aligned}
$$

By Theorem 3.1, we obtain a result analogous to Theorem 2.8.

Corollary 3.2 Let $(P, \leq, ')$ be a poset with a unary operation and $M$ and $R$ defined by (11) and (12), respectively. Then, $\left(P, \leq,^{\prime}, M, R, 0,1\right)$ is a generalized operator residuated poset if it satisfies both conditions (9) and (10).

Acknowledgements Open access funding provided by TU Wien (TUW). The authors thank the anonymous referees for their valuable comments which improved the quality of the paper. Support of the research by ÖAD, Project CZ 02/2019, and support of the research of the first author by IGA, Project PřF 2019 015, is gratefully acknowledged.

\section{Compliance with ethical standards}

Conflict of interest The authors declare that they have no conflict of interest.

Human and animals rights This article does not contain any studies with human participants or animals performed by any of the authors.

Open Access This article is distributed under the terms of the Creative Commons Attribution 4.0 International License (http://creativecomm ons.org/licenses/by/4.0/), which permits unrestricted use, distribution, and reproduction in any medium, provided you give appropriate credit to the original author(s) and the source, provide a link to the Creative Commons license, and indicate if changes were made.

\section{References}

Birkhoff G, von Neumann J (1936) The logic of quantum mechanics. Ann Math 37:823-843

Chajda I, Länger H (2017a) Residuation in orthomodular lattices. Topol Algebra Appl 5:1-5

Chajda I, Länger H (2017b) Orthomodular lattices can be converted into left residuated l-groupoids. Miskolc Math Notes 18:685-689

Chajda I, Länger H (2018) Residuated operators in complemented posets. Asian Eur J Math 11:1850097 (15 pages)

Chajda I, Länger H, Left residuated lattices induced by lattices with a unary operation. Soft Comput (submitted)

Chajda I, Rachůnek J (1989) Forbidden configurations for distributive and modular ordered sets. Order 5:407-423

Galatos N, Jipsen P, Kowalski T, Ono H (2007) Residuated lattices: an algebraic glimpse at substructural logics. Elsevier, Amsterdam

Giuntini R, Ledda A, Paoli F (2016) A new view of effects in a Hilbert space. Studia Logica 104:1145-1177

Giuntini R, Ledda A, Paoli F (2017) On some properties of PBZ*lattices. Int J Theor Phys 56:3895-3911

Husimi K (1937) Studies on the foundation of quantum mechanics I. Proc Phys Math Soc Jpn 19:766-789

Kalman JA (1958) Lattices with involution. Trans Am Math Soc $87: 485-491$

Publisher's Note Springer Nature remains neutral with regard to jurisdictional claims in published maps and institutional affiliations. 